\title{
An elderly woman with an age-old disease
}

\author{
Nicola Mumoli MD, Marco Cei MD
}

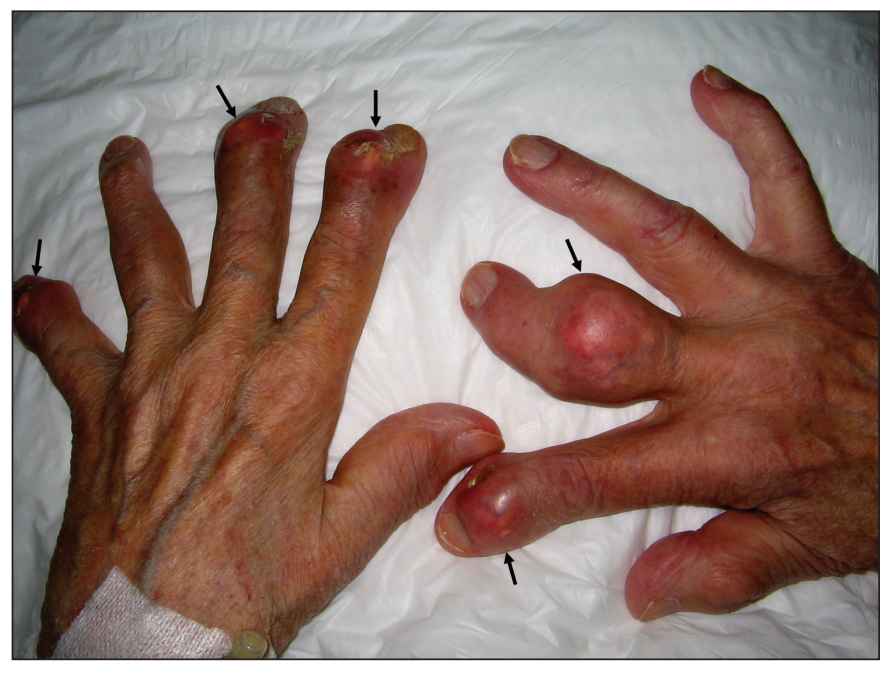

Figure 1: Photograph of the hands of a 104-year-old woman, showing hard, ulcerated and chalky tophi (arrows) on the interphalangeal joints of the second, third and fifth fingers of the left hand and the second and third fingers of the right hand.

\section{A} 104-year-old woman with a 30-year history of gout and hypertension presented for evaluation. Her gout had been treated for many years with allopurinol, and her hypertension was managed with enalapril. She had not taken diuretics and did not drink alcohol. An examination of the patient's hands showed hard, ulcerated and chalky tophi on the interphalangeal joints of the second, third and fifth fingers of the left hand and the second and third fingers of the right hand (Figure 1). Movement of the affected fingers was restricted. The distal interphalangeal joint of the third finger of her right hand was also dislocated (Figure 2). The patient's other joints were normal. Her serum creatinine level was 83 (normal 62-115) $\mu \mathrm{mol} / \mathrm{L}$, and her serum uric acid level was 405 (normal 173-345) $\mu \mathrm{mol} / \mathrm{L}$. A radiograph of the hands (Figure 2) showed erosion of the affected joints and swelling of the surrounding soft tissues. Fine needle aspiration of one tophus showed monosodium urate crystals, which are pathognomonic for gout.

Gout has been a familiar disease since ancient times; evidence of it has even been found in dinosaur remains. ${ }^{1}$ The disease is characterized by a disturbance in the metabolism of urate that causes monosodium urate crystals to be deposited in joints and soft tissues. Other clinical manifestations of gout

From the Department of Internal Medicine, Livorno Hospital, Livorno, Italy

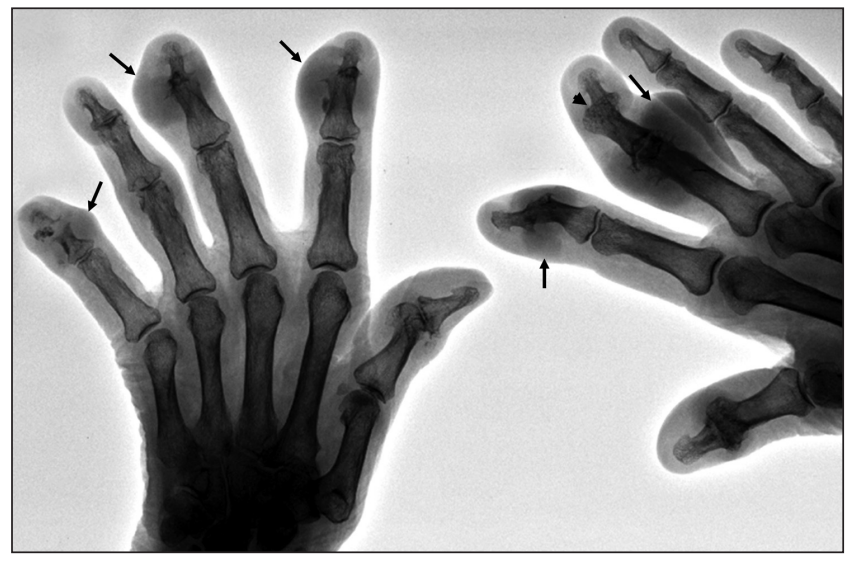

Figure 2: Radiograph of the patient's hands shows joint erosion and soft-tissue swelling (arrows) involving the distal interphalangeal joints of the second, third and fifth fingers of the left hand and the distal interphalangeal joint of the second finger and proximal interphalangeal joint of the third finger of the right hand. The distal interphalangeal joint of the third finger of the right hand is dislocated (arrowhead).

include acute and chronic arthritis, interstitial renal disease and nephrolithiasis. Tophi are caused by an accumulation of deposits of monosodium urate crystals and commonly occur on interphalangeal joints, around the elbow or in the helix of the ear. They are usually painless but may ulcerate and drain white, chalky urate crystals. Tophi usually result from recurrent acute episodes of gout, appearing an average of 12 years after the initial episode.

Gout in elderly patients is often misdiagnosed or identified late in the course of the disease. It tends to have a polyarticular onset involving the hands and is frequently associated with primary renal impairment and use of drugs that reduce the renal excretion of urate (e.g., diuretics and low-dose acetylsalicylic acid). ${ }^{2}$ Many factors (e.g., poor diet, alcohol consumption, nonadherence or intolerance to medication, poor response to treatment) can lead to inadequate control of gout. Our patient's adherence to treatment was poor. As a result, the disease was relentlessly progressive, involving fewer acute episodes and a more chronic, indolent course of disease that included the growth of tophi.

\section{REFERENCES}

1. Rothschild BM, Tanke D, Carpenter K. Tyrannosaurs suffered from gout. Nature 1997;387:357

2. Ene-Stroescu D, Gorbien MJ. Gouty arthritis. A primer on late-onset gout. Geriatrics 2005;60:24-31 